\title{
Critical Evaluation of CrAssphage as a Molecular Marker for Human- Derived Wastewater Contamination in the Aquatic Environment
}

\author{
Kata Farkas ${ }^{1}$ (D) Evelien M. Adriaenssens ${ }^{2,6}$. David I. Walker ${ }^{3}$. James E. McDonald ${ }^{1} \cdot$ Shelagh K. Malham $^{4}$. \\ Davey L. Jones ${ }^{1,5}$
}

Received: 26 November 2018 / Accepted: 18 January 2019 / Published online: 13 February 2019

(c) The Author(s) 2019

\begin{abstract}
The discharge of human-derived wastewater represents a major threat to water quality with the potential for waterborne disease outbreaks mainly associated with enteric viruses. To prevent illnesses, indicators associated with fecal contamination are monitored in polluted areas, however, their prevalence often does not correlate well with viral pathogens. In this study, we used crAssphage, a recently discovered human-specific gut-associated bacteriophage, for the surveillance of wastewater-derived viral contamination. Untreated and treated wastewater, surface water, sediment and mussel samples were collected monthly over 1 year from the Conwy River and estuary (UK) and were analyzed for crAssphage marker by quantitative PCR. This is the first long-term catchment-to-coast scale study of environmental crAssphage concentrations. CrAssphage was detected in all sample types and showed no distinct seasonal pattern. CrAssphage concentrations were $2 \times 10^{5}-10^{9}$ genome copies $(\mathrm{gc}) / \mathrm{L}$ in all untreated wastewater influent and $10^{7}-10^{8} \mathrm{gc} / \mathrm{L}$ in secondary treated effluent samples, $3 \times 10^{3} \mathrm{gc} / \mathrm{L}-3 \times 10^{7} \mathrm{gc} / \mathrm{L}$ in surface water samples ( $94 \%$ positive) and $2 \times 10^{2}-10^{4} \mathrm{gc} / \mathrm{g}$ sediment ( $68 \%$ positive) and mussel digestive tissue (79\% positive). CrAssphage concentrations were $1-5 \log _{10}$ higher than human enteric virus titers (norovirus, sapovirus, adenovirus, polyomavirus). Our results indicate that crAssphage is well suited to tracking human wastewater contamination and pollution risk assessment in aquatic environments.
\end{abstract}

Keywords Wastewater pollution $\cdot$ CrAssphage $\cdot$ Enteric viruses $\cdot$ Shellfish hygiene $\cdot$ qPCR

Electronic supplementary material The online version of this article (https://doi.org/10.1007/s12560-019-09369-1) contains supplementary material, which is available to authorized users.

Kata Farkas

fkata211@gmail.com

1 School of Natural Sciences, Bangor University, Deiniol Road, Bangor, Gwynedd, UK

2 Microbiology Research Group, Institute of Integrative Biology, University of Liverpool, Liverpool, UK

3 Centre for Environment, Fisheries and Aquaculture Science, Weymouth, Dorset, UK

4 School of Ocean Sciences, Bangor University, Menai Bridge, Anglesey, UK

5 UWA School of Agriculture and Environment, University of Western Australia, Crawley, Australia

6 Present Address: Quadram Institute Bioscience, Norwich Research Park, Norwich, UK

\section{Introduction}

Enteric viruses are the most common etiologic agents of gastroenteritis globally. They are discharged with treated and untreated wastewater into the aquatic environment where they are able to persist for long periods of time (Kotwal and Cannon 2014). Hence, these viruses are often responsible for waterborne and foodborne illnesses due to the use of contaminated recreational water, and the consumption of polluted water and shellfish (Radin 2014; Rodríguez-Lázaro et al. 2012). Over 150 human pathogenic viruses, including noroviruses (NoV), sapoviruses ( $\mathrm{SaV})$, rotaviruses, hepatitis $\mathrm{A} / \mathrm{E}$ viruses, adenoviruses ( $\mathrm{AdV})$, enteroviruses and polyomaviruses, have been identified in watercourses (Rodríguez-Lázaro et al. 2012; Tran et al. 2015). As the detection and surveillance of all pathogenic viral strains is not feasible, indicators are often used for tracking wastewater contamination in the environment (Symonds and Breitbart 2015).

The crAssphage sensu stricto genome (RefSeq accession number NC_024711), hereafter referred to as crAssphage, 
was first assembled from human fecal microbiomes within the last 5 years (Dutilh et al. 2014). Further analysis of human metagenomes showed that crAssphage is a highly abundant virus in the human gut, especially in individuals living in industrialized areas (Honap et al. 2018; Stachler and Bibby 2014). Data mining of virome datasets subsequently revealed that there is a diverse group of crAssrelated phages present in the human gut, potentially representing a new family of viruses (Yutin et al. 2017). The first isolated representative of this family has just been discovered, phiCrAss001, infecting the human gut bacterium Bacteroides intestinalis (Shkoporov et al. 2018). Following the discovery of the prevalence of crAss-like phages in the human gut, their usefulness as a tool for source tracking of human fecal pollution was recognized (Stachler and Bibby 2014). Quantitative PCR (qPCR) assays have been developed for the quantification of the crAssphage in stool samples (Cinek et al. 2018; Liang et al. 2018; Stachler et al. 2017). Assay specificity showed great variation, suggesting that some regions of the crAssphage genome may be similar to other bacteriophage genome segments, whereas other regions are indeed human-specific (Stachler et al. 2018). CrAssphage prevalence has been investigated in the aquatic environment and found at high concentrations in untreated wastewater, wastewater-contaminated stream and in stormwater (Ahmed et al. 2018; García-Aljaro et al. 2017; Stachler et al. 2018). The concentration correlated with rainfall frequency in a polluted stream in a 30-day monitoring trial (Stachler et al. 2018). Nonetheless, crAssphage has not been used for long-term monitoring of wastewater-contaminated environments.

The aim of the research described here was to assess the usefulness of a crAssphage marker specifically as an indicator for wastewater-associated viral contamination in the aquatic environment. CrAssphage marker concentrations were monitored in treated and untreated wastewater, riverine and estuarine water, and sediment and shellfish samples collected monthly over 1 year along the Conwy River and estuary, UK. CrAssphage marker concentrations were compared with common enteric viral contaminants, namely NoV genogroups GI and GII (NoVGI and NoVGII), SaV genogroup GI, AdV and JC polyomavirus (JCV).

\section{Materials and Methods}

\section{Sample Types and Process}

We investigated the presence and concentration of crAssphage in nucleic acid extracted from concentrated wastewater, surface water, sediment and shellfish (blue mussel, Mytilus edulis) samples that were known to be contaminated with enteric viruses. The sampling schedule, locations and sample processing are detailed in Farkas et al (2018a) and summarized in Table 1. Thirteen samples were taken of each sample type once per month over a 1-year cycle (August 2016-August 2017).

Table 1 Percentile of virus-positive samples in wastewater (WW), surface water (SW), sediment (Sed) and mussel (SF) samples collected in the Conwy catchment and estuary between August 2016 and August 2017

\begin{tabular}{|c|c|c|c|c|c|c|c|c|}
\hline Sample code & Site & Sample type & NoVGI $(\%)$ & NoVGII (\%) & $\mathrm{SaV}(\%)$ & $\operatorname{AdV}(\%)$ & $\operatorname{JCV}(\%)$ & CrAssphage (\%) \\
\hline GI & Ganol & WW influent & 62 & 69 & 38 & 92 & 100 & 100 \\
\hline BI & Betws-y-Coed & WW influent & 31 & 62 & 15 & 85 & 85 & $100^{\mathrm{a}}$ \\
\hline $\mathrm{BE}$ & Betws-y-Coed & WW effluent (AC) & 31 & 38 & 0 & 77 & 85 & $100^{\mathrm{a}}$ \\
\hline LI & Llanrwst & WW influent & 15 & 54 & 15 & 85 & 85 & $100^{\mathrm{a}}$ \\
\hline LE & Llanrwst & WW effluent (BF) & 31 & 62 & 15 & 92 & 100 & 100 \\
\hline TI & Tal-y-Bont & WW influent & 31 & 62 & 38 & 100 & 92 & $100^{\mathrm{a}}$ \\
\hline TE & Tal-y-Bont & WW effluent (AC) & 45 & 46 & 15 & 92 & 85 & $100^{\mathrm{a}}$ \\
\hline SW1 & Betws-y-Coed-Llugwy River & River water & 15 & 23 & 8 & 85 & 54 & 92 \\
\hline SW2 & Betws-y-Coed-Conwy River & River water & 0 & 8 & 8 & 85 & 54 & $92^{\mathrm{a}}$ \\
\hline SW3 & Llanrwst & Estuarine water & 15 & 8 & 0 & 92 & 92 & 92 \\
\hline SW4 & Conwy & Estuarine water & 8 & 15 & 0 & 92 & 62 & 92 \\
\hline Sed 1 & Deganwy beach & Estuarine sediment & 31 & 15 & 0 & 77 & 46 & 62 \\
\hline Sed2 & Morfa beach & Estuarine sediment & 8 & 0 & 0 & 85 & 46 & $75^{\mathrm{a}}$ \\
\hline Sed4 & Conwy & Estuarine sediment & 15 & 8 & 0 & 85 & 23 & 62 \\
\hline SF1 & Deganwy beach & Mussel & 15 & 15 & 0 & 85 & 31 & 77 \\
\hline SF2 & Morfa beach & Mussel & 23 & 8 & 0 & 85 & 38 & $83^{\mathrm{a}}$ \\
\hline
\end{tabular}

$A C$ activated sludge treatment, $B F$ biofilter; $n=13,{ }^{a} n=12$. NoV GI and GII Norovirus GI and GII, SaV sapovirus, $A d V$ adenovirus and $J C V$ JC polyomavirus data were adopted from Farkas et al. (2018a) 
The water and wastewater samples, $10 \mathrm{~L}$ and $1 \mathrm{~L}$, respectively, were concentrated using tangential flow ultrafiltration followed by beef extract elution and polyethylene glycol (PEG) precipitation (Farkas et al. 2018). Viruses were eluted from $10 \mathrm{~g}$ of sediment using beef extract solution and precipitated with PEG (Farkas et al. 2017). The mussel digestive tissue samples, $2 \mathrm{~g}$ each, were processed as described in the ISO-TS 15216 standard (International Organizatoin for Standardization 2013). Nucleic acids were extracted from the concentrates using the MiniMag NucliSens Nucleic Acid Extraction System (BioMérieux SA, France). Assay efficiency was assessed using mengovirus as a process control, which gave $>10 \%$ recovery values (Farkas et al. 2018).

\section{CrAssphage Quantification}

CrAssphage DNA was quantified using the CPQ_056 TaqMan primers and probe, which have shown great accuracy and little cross-reaction with animal litter (Ahmed et al. 2018; Stachler et al. 2017). The qPCR assays were carried out in a QuantStudio ${ }^{\circledR}$ Flex 6 Real-Time PCR System (Applied Biosystems, USA). The $20 \mu \mathrm{L}$ reaction mix contained $1 \mathrm{x}$ KAPA Probe Force qPCR mix (KAPA Biosystems, USA) with 10 pmol of the forward, $10 \mathrm{pmol}$ of the reverse primers, $5 \mathrm{pmol}$ of the probe, $1 \mu \mathrm{g}$ bovine serum albumin, $50 \mathrm{nM}$ ROX reference dye. The sample volume was $2 \mu \mathrm{L}$ for wastewater influent, $4 \mu \mathrm{L}$ for wastewater effluent and surface water, and $8 \mu \mathrm{L}$ for sediment and mussel samples in each reaction. For quantification, dilution series of a plasmid DNA carrying the target sequence were used. Non-template controls (molecular-grade water) were added to each reaction plate. Amplification was carried out using the following thermal cycling conditions: $98^{\circ} \mathrm{C}$ for $5 \mathrm{~min}$, then 40 cycles of $95^{\circ} \mathrm{C}$ for $15 \mathrm{~s}, 60^{\circ} \mathrm{C}$ for $1 \mathrm{~min}$. The assay efficiency was $90-110 \%$.

\section{Data Analysis}

The viral concentrations were expressed as $\mathrm{gc} / \mathrm{L}$ of wastewater and surface water or gc/g of sediment or mussel digestive tissue. Linear regressions and Spearman's rank correlation coefficients $(r)$ were calculated between viral concentrations using two-tailed 95\% confidence intervals in SigmaPlot 13.0 (Systat Software Inc., US).

\section{Results and Discussion}

\section{CrAssphage qPCR}

The main goal of this study was to evaluate the usefulness of a novel human-associated phage, the crAssphage, as a viral indicator for wastewater contamination. We, therefore, analyzed crAssphage concentrations in wastewater influent and effluent samples released into the river, and in river and estuarine water, sediment and mussel samples contaminated with wastewater, and correlated the concentrations of the crAssphage with enteric viral titres in the samples using the qPCR results. In our study, the non-template controls were negative in each assay suggesting no cross-contamination. The LOD was $2 \mathrm{gc} /$ reaction and samples below that concentration were considered negative. The LOQ was $20 \mathrm{gc} /$ reaction. Dilution of samples did not affect qPCR results indicating no inhibition in the wastewater effluent, sediment or mussel matrices. We utilized a wellestablished primer and probe set that has previously been used for the quantification of crAssphage in stool, wastewater, storm drain outfall and surface water (Ahmed et al. 2018; Stachler et al. 2017, 2018). The qPCR assay has previously shown good human specificity, with little cross-reaction with dog, gull and poultry litter (Ahmed et al. 2018; Stachler et al. 2017). Overall, the qPCR assay used for crAssphage quantification appeared to be accurate and sensitive and hence suitable for the analysis of environmental samples.

\section{CrAssphage Concentrations in Wastewater and in the Aquatic Environment}

All wastewater influent and effluent samples were positive for crAssphage with no seasonal patterns discernible (Table 1; Table S1). In the wastewater influent samples, crAssphage concentrations varied between $2.2 \times 10^{5} \mathrm{gc} / \mathrm{L}$ and $1.2 \times 10^{9} \mathrm{gc} / \mathrm{L}$, with the lowest concentrations observed in the samples collected at Llanrwst (serving approx. 4000 inhabitants) and the highest concentrations in samples derived from the Ganol WWTP (serving approx. 82,000 inhabitants). These concentrations are lower than previously observed in untreated wastewater in Tampa, Florida $\left(10^{9}-10^{10} \mathrm{gc} / \mathrm{L}\right)$ (Ahmed et al. 2018) and in Catalonia, Spain $\left(10^{8}-10^{9} \mathrm{gc} / \mathrm{L}\right)$ (García-Aljaro et al. 2017). The differences in crAssphage concentrations in untreated wastewater may be due to the differences in geographic viral distribution (Stachler and Bibby 2014), WWTP sizes and the level of urbanization, which has been shown to affect crAssphage abundance among inhabitants (Honap et al. 2018).

CrAssphage concentrations in wastewater effluent samples ranged between $1.7 \times 10^{5}$ and $2.0 \times 10^{8} \mathrm{gc} / \mathrm{L}$. In general, we observed a very low (less than $1-\log _{10}$ ) reduction of crAssphage during wastewater treatment at Llanrwst (using biofilter treatment), whereas the concentrations were reduced by up to $2-\log _{10}$ at the Betws-y-Coed and Tal-y-Bont WWTPs (using activated sludge treatment). This suggests that there is a difference in the performance of the different types of wastewater treatment methods. To date, no quantitative studies have assessed the prevalence of crAssphage in treated wastewater.

CrAssphage was detected in $92 \%$ of all river and estuarine water samples (Table 1; Table S1). The concentration 
of crAssphage was approx. 1-3 $\log _{10}$ lower in the surface water relative to the treated wastewater and varied between $3.0 \times 10^{3} \mathrm{gc} / \mathrm{L}$ and $2.5 \times 10^{7} \mathrm{gc} / \mathrm{L}$. The highest concentrations were observed in the SW3 samples $\left(3.0 \times 10^{6} \mathrm{gc} / \mathrm{L}\right.$ on average) derived approximately $200 \mathrm{~m}$ downstream of the Llanrwst wastewater discharge point. These concentrations are consistent with the previously reported crAssphage concentrations in wastewater-polluted surface water $\left(10^{4}-10^{6} \mathrm{gc} / \mathrm{L}\right)$ (Stachler et al. 2018). No seasonal patterns in the crAssphage concentrations in the surface water samples were observed.

The sediment and mussel samples had more negatives than the wastewater and surface water samples (Table 1 and $\mathrm{S} 1)$ with concentrations up to $1.9 \times 10^{4} \mathrm{gc} / \mathrm{g}$ sediment or mussel digestive tissue. No seasonality or significant differences were observed among the sediment and mussel sample
crAssphage concentrations. Detection of crAssphage in sediment or in mussel samples has not previously been reported.

\section{Comparison of Enteric Virus and CrAssphage Concentrations in Different Sample Types}

CrAssphage concentrations in wastewater, surface water, sediment and mussel samples were compared with the human-specific NoVGI, NoVGII, SaV, AdV and JCV concentrations of the same samples, which were determined in a previous study (Farkas et al. 2018). The enteric viruses were less prevalent and were present at lower concentrations than crAssphage. The virus-positive wastewater samples, both influent and effluent wastewater samples, had approx. $2 \log _{10}$ higher crAssphage than enteric virus titers (Fig. 1).
Fig. 1 Box plot showing the median concentration (minmax) of crAssphage (blue), human adenovirus ( $\mathrm{AdV}$; green), JC polyomavirus (JCV; gray) norovirus GI (NoVGI; purple) and GII (NoVGII, yellow) and sapovirus (SaV; red) determined in (A) wastewater influent (GI, BI, LI and TI) and effluent (BE, LE, TE) and in (B) surface water (SW1-4), sediment (Sed1, 2, 4) and mussel (SF1, 2). Sample concentration below the limit of quantification was considered $100 \mathrm{gc} / \mathrm{l}$ or $\mathrm{gc} / \mathrm{g}$. Circles: outliers, starts extreme outliers
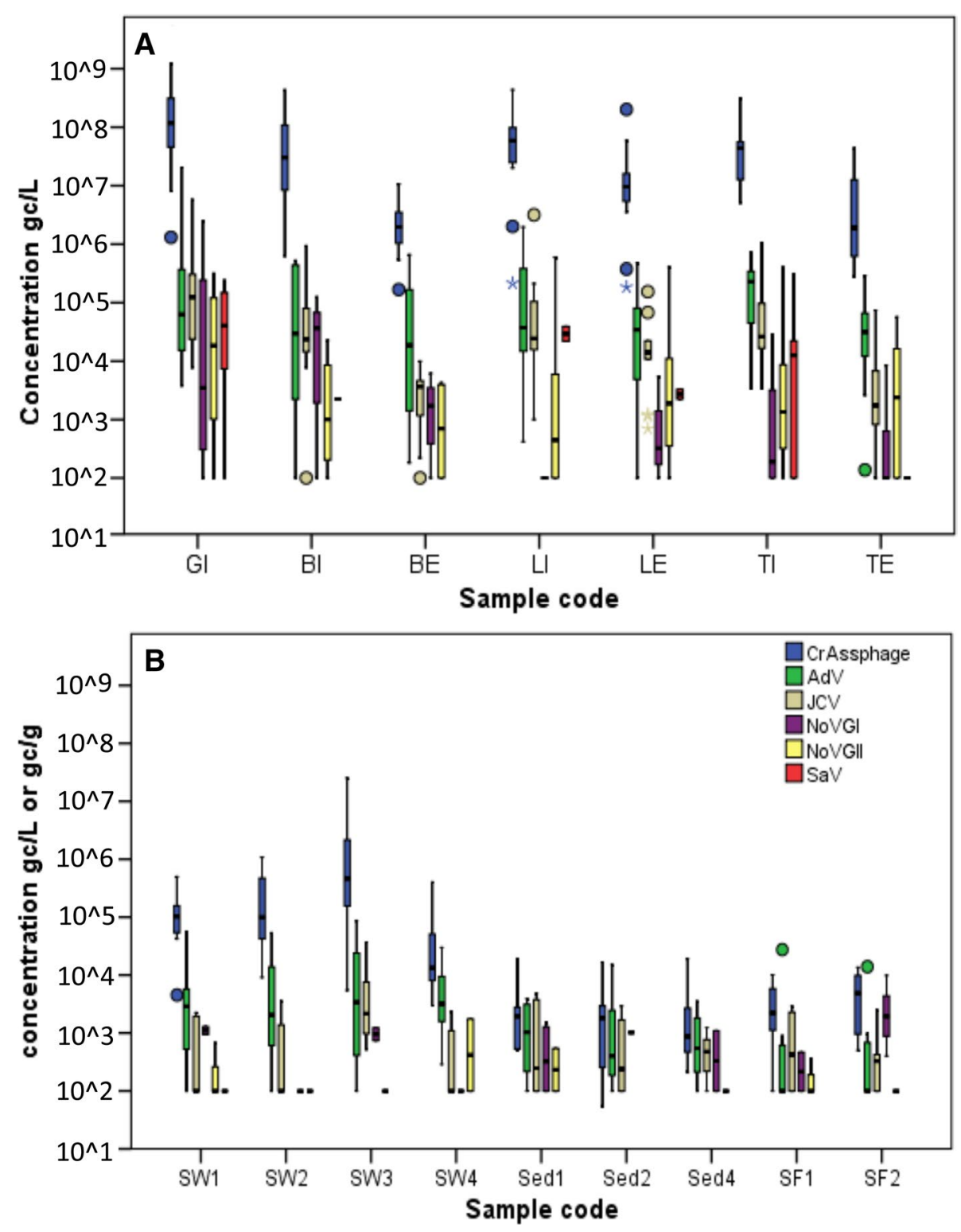
Table 2 Spearman correlation between crAssphage and human enteric virus titers

\begin{tabular}{lllr}
\hline Sample type & Viruses & \multicolumn{2}{r}{$p$} \\
\hline Wastewater influent & CrAssphage-AdV & $0.31^{*}$ & 0.03 \\
& CrAssphage-JCV & $0.50^{* * *}$ & $<0.01$ \\
& CrAssphage-NoVGI & 0.26 & 0.07 \\
& CrAssphage-NoVGII & $0.34^{*}$ & 0.02 \\
& CrAssphage-SaV & 0.265 & 0.07 \\
Wastewater effluent & CrAssphage-AdV & 0.13 & 0.45 \\
& CrAssphage-JCV & $0.67 * * *$ & $<0.01$ \\
& CrAssphage-NoVGI & 0.20 & 0.23 \\
& CrAssphage-NoVGII & $0.43^{* *}$ & $<0.01$ \\
Surface water & CrAssphage-SaV & 0.21 & 0.21 \\
& CrAssphage-AdV & -0.02 & 0.88 \\
& CrAssphage-JCV & $0.49 * * *$ & $<0.01$ \\
& CrAssphage-NoVGI & 0.08 & 0.55 \\
& CrAssphage-NoVGII & 0.07 & 0.64 \\
Sediment & CrAssphage-SaV & -0.07 & 0.63 \\
& CrAssphage-AdV & $0.37 *$ & 0.02 \\
& CrAssphage-JCV & 0.09 & 0.61 \\
& CrAssphage-NoVGI & -0.08 & 0.63 \\
& CrAssphage-NoVGII & 0.24 & 0.14 \\
\hline
\end{tabular}

$R$ : correlation coefficient. Further data are shown in Supplementary Table 2. $* p<0.05 ; * * p<0.01 ; * * * p<0.001$. No SaV was detected in the sediment

A weak correlation $(r<0.5)$ among viral titers was observed in wastewater influent except for the crAssphage/JCV and the $\mathrm{AdV} / \mathrm{SaV}$ pairs, which showed moderate correlation $(r=0.50$ and 0.55 ; Table 2 and S2). The reduction rate of all tested viruses in the WWTPs were very similar, resulting in approximately $1 \log _{10}$ reduction during biofilter treatment and 1-2 $\log _{10}$ reduction during activated sludge treatment. Similar reduction trends have been reported in other studies on enteric virus and coliphage titers in activated sludge and biofilter-treated wastewater (Campos et al. 2016; Hewitt et al. 2011; Kitajima et al. 2014; Sidhu et al. 2017). In wastewater effluent, strong correlation was noted for crAssphage/JCV $(r=0.67$; Table 2). The crAssphage and JCV showed a linear correlation in the untreated wastewater samples, however, such correlation was only observed in the wastewater effluents that underwent biofilter treatment $\left(R^{2}=0.81\right.$; Figure $\left.\mathrm{S} 1\right)$.

In the surface water samples, crAssphage was more prevalent than the enteric viruses. Only AdV was detected in a similar number of samples to crAssphage (85-92\%), whereas the detection rates of the JCV (54-92\%) and the NoVs and SaV (0-23\%) were lower (Table 1). The concentration of crAssphage was 1-2 $\log _{10}$ higher than the concentration of the AdV and JCV, and 2-5 $\log _{10}$ higher than that observed for NoVs and $\mathrm{SaV}$ (Fig. 1). In the sediment and mussel samples, the detection rate of crAssphage (62-75\%) was slightly lower than the detection rate of AdV (77-85\%) but higher than observed for the JCV (23-46\%) and NoVs (0-31\%; Table 1). The concentration of crAssphage in sediment was similar to the concentration of AdV and JCV in sediment and slightly higher in mussel (Fig. 1). Weak or no correlation was observed among viruses in surface water, sediment and shellfish samples (Table S2). A linear correlation between crAssphage and JCV was only observed in the highly polluted surface water sample (SW3), where the highest viral titers were observed (Figure S1). The lack of correlation between viral titers is probably due to the low copy numbers and the low number of positive samples.

\section{Environmental Implications and Future Research}

In this study, the concentration of crAssphage in wastewater and in wastewater-contaminated environments was tracked over a period of 1 year for the first time. Similar to AdV and JCV, which are considered markers for human wastewater pollution (Rachmadi et al. 2016; Rames et al. 2016), crAssphage incidence displayed no seasonal patterns and was prevalent in all sample types. These findings suggest that the use of crAssphage as a fecal contamination indicator enables the assessment of pollutant transport. This was the first time crAssphage detection was addressed in sediment and in bivalve shellfish. CrAssphage was frequently detected in mussel and sediment, collected at the wastewater-contaminated areas, demonstrating that crAssphage may be a suitable fecal pollution indicator in those matrices.

Future research should explore the usefulness of qPCR assays targeting different regions of the crAssphage genome to enhance specificity, explore human specificity and exploit the recently discovered new crAss-like genomes to identify geographic differences in diversity and assess correlations between the concentrations of crAss-like phages and a wide range of enteric viruses and other viral and bacterial indicators. The prevalence of crAssphage in waters not affected by wastewater contamination also needs to be established. Furthermore, with the very recent discovery of a crAss-like phage isolate (Guerin et al. 2018) and the successful in vitro culturing of that strain (Shkoporov et al. 2018) future approaches should be focused on comparative description of crAssphage, F-RNA phage and AdV/JCV infectivity, survival and persistence in wastewater and in the aquatic environment, including surface and groundwater, and in bivalve shellfish and fresh produce. Once established, crAssphage may be a suitable marker for routine bivalve shellfish testing in commercial fisheries.

Acknowledgements This work was supported by the Natural Environment Research Council (NERC) and the Food Standards Agency (FSA) under the Environmental Microbiology and Human Health (EMHH) Programme (NE/M010996/1). We thank Gordon Steffen (Welsh Water, UK) for providing wastewater samples and Prof Alan 
McCarthy (University of Liverpool) for his helpful review comments on the manuscript.

\section{Compliance with Ethical Standards}

Conflict of interest The authors declare no conflict of interest.

Open Access This article is distributed under the terms of the Creative Commons Attribution 4.0 International License (http://creativeco mmons.org/licenses/by/4.0/), which permits unrestricted use, distribution, and reproduction in any medium, provided you give appropriate credit to the original author(s) and the source, provide a link to the Creative Commons license, and indicate if changes were made.

\section{References}

Ahmed, W., Lobos, A., Senkbeil, J., Peraud, J., Gallard, J., \& Harwood, V. J. (2018). Evaluation of the novel crAssphage marker for sewage pollution tracking in storm drain outfalls in Tampa, Florida. Water Research, 131, 142-150. https://doi.org/10.1016/j.watre s.2017.12.011.

Ahmed, W., Payyappat, S., Cassidy, M., Besley, C., \& Power, K. (2018). Novel crAssphage marker genes ascertain sewage pollution in a recreational lake receiving urban stormwater runoff. Water Research, 145, 769-778. https://doi.org/10.1016/j.watre s.2018.08.049.

Ahmed, W., Zhang, Q., Lobos, A., Senkbeil, J., Sadowsky, M. J., Harwood, V. J., et al. (2018). Precipitation influences pathogenic bacteria and antibiotic resistance gene abundance in storm drain outfalls in coastal sub-tropical waters. Environment International, 116(March), 308-318. https://doi.org/10.1016/j.envin t.2018.04.005.

Campos, C. J. A., Avant, J., Lowther, J., Till, D., \& Lees, D. N. (2016). Human norovirus in untreated sewage and effluents from primary, secondary and tertiary treatment processes. Water Research, 103, 224-232. https://doi.org/10.1016/j.watres.2016.07.045.

Cinek, O., Mazankova, K., Kramna, L., Odeh, R., Alassaf, A., Ibekwe, M. A. U., et al. (2018). Quantitative CrAssphage real-time PCR assay derived from data of multiple geographically distant populations. Journal of Medical Virology, 90(4), 767-771. https://doi. org/10.1002/jmv.25012.

Dutilh, B. E., Cassman, N., McNair, K., Sanchez, S. E., Silva, G. G. Z., Boling, L., et al. (2014). A highly abundant bacteriophage discovered in the unknown sequences of human faecal metagenomes. Nature Communications, 5, 1-11. https://doi.org/10.1038/ ncomms5498.

Farkas, K., Cooper, D. M., McDonald, J. E., Malham, S. K., de Rougemont, A., Jones, D. L., et al. (2018). Seasonal and spatial dynamics of enteric viruses in wastewater and in riverine and estuarine receiving waters. Science of the Total Environment, 634, 1174 1183. https://doi.org/10.1016/j.scitotenv.2018.04.038.

Farkas, K., Hassard, F., McDonald, J. E., Malham, S. K., \& Jones, D. L. (2017). Evaluation of molecular methods for the detection and quantification of pathogen-derived nucleic acids in sediment. Frontiers in Microbiology, 8, 53. https://doi.org/10.3389/fmicb .2017 .00053 .

Farkas, K., Mcdonald, J. E., Malham, S. K., \& Jones, D. L. (2018). Two-step concentration of complex water samples for the detection of viruses. Methods and Protocols, 1(3), 35. https://doi. org/10.3390/mps1030035.

García-Aljaro, C., Ballesté, E., Muniesa, M., \& Jofre, J. (2017). Determination of crAssphage in water samples and applicability for tracking human faecal pollution. Microbial Biotechnology, 10(6), 1775-1780. https://doi.org/10.1111/1751-7915.12841.

Guerin, E., Shkoporov, A., Stockdale, S. R., Clooney, A. G., Ryan, J., Draper, L. A., et al. (2018). Biology and taxonomy of crAsslike bacteriophages, the most abundant virus in the human gut. bioRxiv. https://doi.org/10.1101/295642.

Hewitt, J., Leonard, M., Greening, G. E., \& Lewis, G. D. (2011). Influence of wastewater treatment process and the population size on human virus profiles in wastewater. Water Research, 45(18), 6267-6276. https://doi.org/10.1016/j.watres.2011.09.029.

Honap, T. P., Sankaranarayanan, K., Schnorr, S. L., Ozga, A. T., Warinner, C., \& Lewis, C. M. (2018). Biogeographic study of human gut associated crAssphage suggests impacts from industrialization and recent expansion. bioRxiv. https://doi.org/10.1101/384677.

International Organizatoin for Standardization. (2013). International Organization for Standardization ISO/TS 15216-2:2013-Microbiology of food and animal feed-Horizontal method for determination of hepatitis A virus and norovirus in food using real-time RT-PCR-Part 2: Method for qualitative detection.

Kitajima, M., Iker, B. C., Pepper, I. L., \& Gerba, C. P. (2014). Relative abundance and treatment reduction of viruses during wastewater treatment processes-Identification of potential viral indicators. Science of the Total Environment, 488, 290-296. https://doi. org/10.1016/j.scitotenv.2014.04.087.

Kotwal, G., \& Cannon, J. L. (2014). Environmental persistence and transfer of enteric viruses. Current Opinion in Virology, 4, 37-43. https://doi.org/10.1016/j.coviro.2013.12.003.

Liang, Y., Jin, X., Huang, Y., \& Chen, S. (2018). Development and application of a real-time polymerase chain reaction assay for detection of a novel gut bacteriophage (crAssphage). Journal of Medical Virology, 90(3), 464-468. https://doi.org/10.1002/ jmv.24974.

Rachmadi, A. T., Torrey, J. R., \& Kitajima, M. (2016). Human polyomavirus: Advantages and limitations as a human-specific viral marker in aquatic environments. Water Research, 105, 456-469. https://doi.org/10.1016/j.watres.2016.09.010.

Radin, D. (2014). New trends in food-and waterborne viral outbreaks. Archives of Biological Sciences, 66(1), 1-9. https://doi. org/10.2298/ABS1401001R.

Rames, E., Roiko, A., Stratton, H., \& Macdonald, J. (2016). Technical aspects of using human adenovirus as a viral water quality indicator. Water Research, 96, 308-326. https://doi.org/10.1016/j.watre s.2016.03.042.

Rodríguez-Lázaro, D., Cook, N., Ruggeri, F. M., Sellwood, J., Nasser, A., Nascimento, M. S. J., et al. (2012). Virus hazards from food, water and other contaminated environments. FEMS Microbiology Reviews, 36(4), 786-814. https://doi.org/10.111 1/j.1574-6976.2011.00306.x.

Shkoporov, A. N., Khokhlova, E. V., Fitzgerald, C. B., Stockdale, S. R., Draper, L. A., Ross, R. P., \& Hill, C. (2018). ФCrAss001 represents the most abundant bacteriophage family in the human gut and infects Bacteroides intestinalis. Nature Communications, 9(1), 4781. https://doi.org/10.1038/s41467-018-07225-7.

Sidhu, J. P. S., Ahmed, W., Palmer, A., Smith, K., Hodgers, L., \& Toze, S. (2017). Optimization of sampling strategy to determine pathogen removal efficacy of activated sludge treatment plant. Environmental Science and Pollution Research, 24(23), 19001-19010. https://doi.org/10.1007/s11356-017-9557-5.

Sidhu, J. P. S., Sena, K., Hodgers, L., Palmer, A., \& Toze, S. (2017). Comparative enteric viruses and coliphage removal during wastewater treatment processes in a sub-tropical environment. Science of the Total Environment, 616, 669-677. https://doi.org/10.1016/j. scitotenv.2017.10.265.

Stachler, E., Akyon, B., De Carvalho, A., Ference, N., C., \& Bibby, K. (2018). Correlation of crAssphage-based qPCR markers with culturable and molecular indicators of human fecal pollution in 
an impacted urban watershed. Environmental Science and Technology, 52, 7505-7512. https://doi.org/10.1021/acs.est.8b00638.

Stachler, E., \& Bibby, K. (2014). Metagenomic evaluation of the highly abundant human gut bacteriophage CrAssphage for source tracking of human fecal pollution. Environmental Science \& Technology Letters, 1, 405-409. https://doi.org/10.1021/ez500266s.

Stachler, E., Kelty, C., Sivaganesan, M., Li, X., Bibby, K., \& Shanks, O. C. (2017). Quantitative CrAssphage PCR assays for human fecal pollution measurement. Environmental Science and Technology, 51(16), 9146-9154. https://doi.org/10.1021/acs.est.7b02703.

Symonds, E. M., \& Breitbart, M. (2015). Affordable enteric virus detection techniques are needed to support changing paradigms in water quality management. Clean Soil Air Water, 43(1), 8-12. https://doi.org/10.1002/clen.201400235.

Tran, N. H., Gin, K. Y. H., \& Ngo, H. H. (2015). Fecal pollution source tracking toolbox for identification, evaluation and characterization of fecal contamination in receiving urban surface waters and groundwater. Science of the Total Environment. https://doi. org/10.1016/j.scitotenv.2015.07.155.

Yutin, N., Makarova, K. S., Gussow, A. B., Krupovic, M., \& Segall, A. (2017). Discovery of an expansive family of bacteriophages that includes the most abundant viruses from the human gut. Nature Microbiology, 1-9. https://doi.org/10.1038/s41564-017-0053-y.

Publisher's Note Springer Nature remains neutral with regard to jurisdictional claims in published maps and institutional affiliations. 